\begin{tabular}{|l|l|}
\hline IEEE & IEEE Transactions on Geoscience \\
and Remote Sensing
\end{tabular}

\title{
Absolute radiometric calibration of narrow-swath imaging sensors with reference to non-coincident wide-swath sensors
}

\begin{tabular}{|r|l|}
\hline Journal: & Transactions on Geoscience and Remote Sensing \\
\hline Manuscript ID: & TGRS-2012-00193 \\
\hline Manuscript Type: & Inter-Calibration of Satellite Instruments Special Issue \\
\hline Date Submitted by the Author: & 21 -Feb-2012 \\
\hline Complete List of Authors: & $\begin{array}{l}\text { McCorkel, Joel; NASA, Biospheric Sciences } \\
\text { Thome, Kurt; NASA, Biospheric Sciences } \\
\text { Lockwood, Ronald; MIT Lincoln Laboratory, Group 97 - Sensors Technology } \\
\text { and Systems Applications }\end{array}$ \\
\hline Keywords: & Calibration, Remote sensing \\
\hline
\end{tabular}


Absolute radiometric calibration of narrow-swath imaging sensors with reference to non-coincident wide-swath sensors

Joel McCorkel, Kurtis Thome, Ronald Lockwood

Sciences and Exploration Directorate, NASA/GSFC, Greenbelt, MD 20771, USA

\begin{abstract}
An inter-calibration method is developed to provide absolute radiometric calibration of narrow-swath imaging sensors with reference to non-coincident wide-swath sensors. The method predicts at-sensor radiance using non-coincident imagery from the reference sensor and knowledge of spectral reflectance of the test site. The imagery of the reference sensor is restricted to acquisitions that provide similar view and solar illumination geometry to reduce uncertainties due to directional reflectance effects. Spectral reflectance of the test site is found with a simple iterative radiative transfer method using radiance values of a well-understood wide-swath sensor and spectral shape information based on historical ground-based measurements. At-sensor radiance is calculated for the narrow-swath sensor using this spectral reflectance and atmospheric parameters that are also based on historical in situ measurements. Results of the inter-calibration method show agreement on the $2-5 \%$ level in most spectral regions with the vicarious calibration technique relying on coincident ground-based measurements referred to as the reflectance-based approach. While the variability of the inter-calibration method based on non-coincident image pairs is significantly larger, results are consistent with techniques relying on in situ measurements. The method is also insensitive to spectral differences between the sensors by transferring to surface spectral reflectance prior to prediction of at-sensor radiance. The utility of this inter-calibration method is made clear by its flexibility to utilize image pairings with acquisition dates differing in excess of 30 days allowing frequent absolute calibration comparisons between wide- and narrow-swath sensors.
\end{abstract}

\title{
I. Introduction
}

Earth-observing satellite sensors provide measurements for a wide range of climatic and environmental studies. It is critical that these sensors remain calibrated in order to achieve synergy among coexisting sensors and legacy among generations of sensors. There are several methods of radiometric calibration of satellite sensors including preflight, on-board instrumentation, lunar, or solar illumination, and methods that use terrestrial scenes acquired on-orbit. Each method has its own strengths and weaknesses. Preflight characterization is the most thorough but it is often difficult to 
create test apparatus that exactly emulate operational conditions and anticipate how the senor may be affected by launch and the space environment (Barnes et al., 2001). Many sensors, including Hyperion, have on-board calibrators that provide valuable information about sensor behavior such as detector-to-detector variability and trends in sensor health. However, on-board calibrators will never be able to provide an absolute calibration that is more accurate than the preflight characterization.

Several techniques have been developed to characterize sensors vicariously and independently from prelaunch and on-board calibrators including repeated lunar acquisitions, comparisons to other sensors, and comparisons to ground-based measurements. One method of vicarious validation of at-sensor radiance is the reflectance-based approach, successfully implemented by several research groups and applied to dozens of Earth-observing sensors (Vane et al, 1993; Thome, 2001; Thome, 2004a,b; Arai et al, 2005). This method relies on in situ measurements that characterize surface reflectance and atmospheric properties of a test site for input to a radiative transfer model to predict at-sensor radiance. These values are then compared with corresponding measurements of the airborne or spaced-based sensor.

One issue with the reflectance-based approach is that it requires collection of the ground data coincident with the view of the site by the sensor being calibrated. Such an approach is problematic when the goal is to calibrate more than a few sensors. Relying on satellite-based sensors to characterize the site or a test site that is invariant with time provides many more opportunities for radiometric calibration through cross-calibration.

Ideally, the data from both sensors would be coincident in time with identical view and solar geometries. Teillet et al. (2001b) developed a variation of the approach to 
account for small changes in view and solar geometry. In this case, the aircraft sensor is used to derive the surface reflectance of a test site both spatially and spectrally (Teillet et al., 2001b).

Simplification of the cross-calibration problem is achieved by limiting the data sets to nearly coincident views of the same test site. The simultaneous nadir overpass (SNO) method is such an approach (Heidinger et al., 2002; Wu et al., 2008). The method obtains the largest number of coincident views near the polar regions for typical sunsynchronous, near-polar orbits. Such overlapping data sets limit the approach to spectral regions for which the radiance from snow and ice can be well predicted because even sensors under comparison sharing similar acquisition times and geometries will differ in spectral coverage exacerbating the calibration transfer (Trischenko et al., 2002; Teillet et al., 2007).

A drawback to the above methods is that one must limit the intercomparisons to geographic regions near the poles where significant overlaps occur or must wait for fortuitous overlaps at other locations. Overlaps are more prevalent between large swath sensors, though they require corrections for differing sun and view angles. The difficulty remains for narrow-swath sensors for which overlapping opportunities may be limited. This work presents a method to transfer the radiometric calibration from a wide-swath sensor to any other sensor. A width-swath sensor has the advantage of sampling the entire Earth's surface nearly every day. This means that most other sensors with varying swath widths and duty cycles must have near-overlapping imagery with varying degrees of similarity between acquisition time and geometry. The wide-swath sensors chosen for this work are MODIS, both on the Terra and Aqua platforms, due to their remarkably 
accurate radiometric calibration (Xiong et al. 2003; Xiong and Barnes, 2006). Terra was launched in December 1999 with a 10:30 am equatorial crossing time and Aqua was launched in May 2002 with a 1:30 pm equatorial crossing time. Terra is commonly known as the 'AM' satellite and Aqua, the 'PM' satellite due to their equatorial crossing times. MODIS has spatial resolutions of 250, 500, and $1000 \mathrm{~m}$, depending on the spectral band, covering a swath width of $2330 \mathrm{~km}$. The MODIS sensors have a total of 36 bands, 19 of which are within the solar reflective region of the electromagnetic spectrum.

Hyperion is a convenient test case for a sensor to calibrate with this method. First, it is an on-demand sensor with a narrow swath of $7.5 \mathrm{~km}$ showing that this method can be used with nearly any sensor. Second, Hyperion is an imaging spectrometer with spectral channels covering the solar reflective region with about 10-nm sampling and 10-11 nm bandwidths allowing this method to be tested with the same spectral fidelity. Lastly, past work has provided a well-understood radiometric characterization of Hyperion that these and future results can be held against (Biggar et al., 2003, McCorkel et al., In review). Hyperion is aboard the Earth Observing 1 spacecraft launched in November 2000, providing significant lifetime overlap with both MODIS sensors.

The next section describes the test site used for this study and past work that will serve as a baseline to assess the reliability of this new method. The past work uses the reflectance-based approach of vicarious calibration to characterize Hyperion. The third section describes the inter-calibration method developed in this work. The method uses the well-understood radiometric calibration of a wide-swath sensor, MODIS, in conjunction with a mean reflectance curve of historical in situ measurements to predict the at-sensor radiance of the test case sensor, Hyperion. The accuracy and variability of 
this method is tested with image dates matching those used in the reflectance-based approach. Further results test the flexibility of this method by using non-coincident image pairs.

\section{Test site description}

Desirable test site properties for the reflectance-based approach as well as other calibration methods include high-reflectance, spatially uniform over large areas, and high in elevation (Scott et al., 1996). The overarching idea behind these characteristics is to get closer to a more ideal case of zero atmosphere by maximizing the signal due to directly reflected solar irradiance. A bright test site with a reflectance with 0.3 or greater maximizes signal component due to directly reflected solar irradiance for most spectral regions. Spatially uniform sites reduce concerns such as registration and adjacency effects seen in some radiative transfer studies when the surface reflectance surrounding the test site is different than that of the test site. Test sites at high elevations have less atmospheric aerosols therefore errors associated with their characterization have less effect. Other desirable test site characteristics are near lambertian reflectance properties and temporally stability. A lambertian site reduces effects due to solar and view geometries. A temporally stable site allows consistency between day-to-day, season-toseason, and year-to-year studies. Lastly, logistics and cost of traveling to a test site with an assortment of personnel and instrumentation control the locality of the test site. The Railroad Valley Playa (RVPN), a large desert basin in Nevada, satisfactorily satisfies the 
criteria described above and is selected for this work. The RVPN test site is centered near $38.497^{\circ} \mathrm{N}, 115.690^{\circ} \mathrm{W}$ and is $1438 \mathrm{~m}$ above sea level.

Over the 2000-2009 period, there have been hundreds of field campaigns that characterize several test sites in the southwestern United States with RVPN being one of the most regularly visited. The atmospheric and surface reflectance data recorded on these campaigns amount to a vast library that spreads over varying seasons and surface conditions. Reflectance of a test site is a function of a multitude of factors including solar illumination angle, soil moisture, and atmospheric conditions. This makes it difficult to predict the reflectance for any given time and hence the need for measurements during the time of sensor overpass (Thome et al., 2004b; Thome et al., 2008).

The historical library of RVPN surface reflectance shows substantial scatter over time and illumination angle, but inspection of this scatter spectrally indicates flat offsets from one day to the next. Consider as an example a collection of nine years of reflectance data of a $1-\mathrm{km}$ by $1-\mathrm{km}$ area of RVPN that is characterized for the reflectance-based calibration of MODIS shown in Figure 1. Each of the 51 thin lines represents reflectance data collected during the reflectance-based approach of calibrating Terra or Aqua MODIS (Thome et al., 2004). Similar results are obtained when considering the larger number of data sets collected for smaller-sized footprints. The effects seen in the figure for spectral regions around $1350 \mathrm{~nm}$ and $1800 \mathrm{~nm}$ are due to strong absorption due to water vapor and therefore measurements have very low signal-to-noise ratio. The thick black line in Figure 1 shows the mean of all 51 reflectance data.

\section{Inter-calibration method}


A method is developed to radiometrically characterize all of the channels of an imaging spectrometer using an inter-calibration scheme. As mentioned above, intercalibration of space-based Earth-observing sensors is a well-documented method of radiometric calibration (Teillet et al., 1990; Cabot et al., 2000; O'Brien and Mitchell, 2001; Teillet et al., 2001a; Thome et al., 2003; Chander et al., 2004; Nguyen et al., 2004; Teillet et al., 2006; Teillet et al., 2007). Most efforts compare temporal trends of various sensors using frequent acquisitions of a near-ideal test site. These methods only provide temporal change relative to each other and the test site itself where other efforts use spectral knowledge of the sensor to make absolute radiometric comparisons. This work uses a well-understood multispectral sensor and a well-known test site to predict a continuous spectrum of at-sensor radiance suitable to characterize a variety of sensors including an imaging spectrometer as used for a test case here. The first part of this section describes the inter-calibration scheme and how it is applied in this work. Next, image pair selection based on acquisition time and geometry is discussed. The third part presents a simple method to derive a continuous reflectance spectrum using MODIS radiance and the last part describes how it is used to find a calibration for Hyperion.

\section{A. Inter-calibration scenarios}

One approach to inter-calibration makes use of spatially overlapping images from the sensors under study. The sensors each have their own orbit, and, therefore their imagery will be acquired with unique view and solar illumination geometries. The ideal 
case for inter-calibration is when the sensors are on the same spacecraft, so as to acquire imagery under identical conditions such as the case for ASTER and Terra MODIS. A slightly less ideal case is when two sensors are in the same orbit, but with a slight temporal difference. For example, before arriving to its destination orbit, Landsat 7 was placed in a tandem orbit with Landsat 5 allowing for the inter-calibration of the Landsat 7 Enhanced Thematic Mapper Plus (ETM+) and Landsat 5 Thematic Mapper (TM) to establish calibration continuity (Teillet et al. 2001a, Teillet et al., 2006). Similar work has been done for ETM+ and Terra MODIS, which follows ETM+ by approximately 30 minutes (Thome et al., 2003). Any difference in acquisition time or date increases uncertainties due to changes in surface and atmospheric conditions affecting the radiance incident at the sensors. The effects of the atmosphere and the surface on the calibration are further exacerbated when the sensors view the same area at different view angles.

This work studies inter-calibration results for two of the above-mentioned scenarios: 1) the sensors have the same geometry and nearly coincident image acquisitions; and 2) the sensors have the nearly the same geometry but different acquisition dates. Studying both scenarios with the same sensor pair is possible because Hyperion's orbit preceded MODIS by 40 minutes early in its mission, but experienced an orbit change in mid-2005, after which only non-coincident comparisons were typical. These two scenarios can be labeled as coincident and non-coincident, referring to the image acquisition time and geometry. The coincident case, as well as results from the reflectance based approach, is held as a baseline for comparing the non-coincident case. Application of the non-coincident case is far more general since not nearly as many sensors fall under the coincident case. The inter-calibration method used for both cases is 
nearly identical, the only difference being that the non-coincident case requires a search of image pairs from the two sensors that have matching geometries.

\section{B. Image pair selection}

Hyperion-MODIS image pairs are selected according to the inter-calibration scenarios discussed above. Image pair selection for the coincident case is straightforward; images acquired on the same date, which have overlapping spatial locations, qualify. The spatial locations are narrowed to the test sites discussed in the next section. Image pair selection for the non-coincident case requires consideration of temporal and geometrical compatibility.

Temporal matching is important due to the variability inherent to atmospheric conditions. This is most easily accounted for through use of a large number of data sets over which outliers such as cloudy days are discarded and smaller atmospheric effects such as variable ozone and water vapor will average out. Differences in imaging geometry have a larger impact on the total radiance seen at the sensor due to the fact that the surface reflectance is a function of view and illumination angles. This variability in reflectance is typically characterized with a bidirectional reflectance distribution function (BRDF). Many surfaces have a BRDF with complicated structure such as vegetation and urban environments or those with a strong specular component such as water and snow. The test sites used for reflectance-based and inter-calibration methods are much simpler in this regard and are assumed to be Lambertian for which BRDF is constant. Effort is given to reduce uncertainties due to variability of surface BRDF by considering only 
similar geometries. The reflectance-based approach relies on measuring surface reflectance close in time to the sensor overpass to minimize uncertainties caused by differing illumination angles between in situ measurements and the satellite imager. The coincident case of inter-calibration has nearly identical viewing geometries and only small differences in illumination angles. The non-coincident scenario relies on selecting image pairs that share similar geometries to reduce BRDF effects.

Processing routines are developed for this work that find Hyperion-MODIS image pairs that share similar view and solar geometry. Inputs to this routine are the library of images from both sensors, a maximum angular difference of the geometries between the two sensors, and the maximum temporal difference between the acquisition dates of the image pair. Restricting the geometry difference between an image pair reduces uncertainties induced by the lack of knowledge about surface BRDF. Restricting the temporal difference between the image pair may reduce the effect of differing atmospheric conditions. However, increased restrictions placed on these parameters result in fewer image pairs satisfying the geometry requirements.

\section{Deriving continuous reflectance spectrum}

A challenge of this work is predicting a continuous spectrum of at-sensor radiance when radiance at discrete spectral positions is known. This is achieved by deriving surface reflectance using a combination of data consisting of multispectral information from MODIS and prior knowledge of the spectral structure of the surface. The RVPN test site has the advantage of nearly a decade of in situ measurements as discussed in the 
previous section and the mean of these measurements is scaled to best fit the MODIS radiance values for each image pair.

A simple iterative model is used to predict multispectral reflectance of the RVPN test site so that the most appropriate scaling of the reflectance curve can be found. Atsensor radiance for an Earth viewing sensor is a sum of different sources: surface reflected radiance and atmospherically scattered radiance. Surface reflected radiance is further specified as a sum of light directly transmitted to and from the surface and reflected downwelling diffuse light scattered by the atmosphere. This sum is represented by Equation 1 as

$$
L_{\text {total }}=L_{\text {direct }}+L_{\text {diffuse }}+L_{\text {sky }} \quad \text { Eqn. } 1
$$

where $\mathrm{L}_{\text {total }}$ is at-sensor radiance, $\mathrm{L}_{\mathrm{direct}}$ is directly transmitted surface reflected radiance, $\mathrm{L}_{\mathrm{diffuse}}$ is diffuse atmospheric reflected radiance, and $\mathrm{L}_{\mathrm{sky}}$ is upwelling atmospherically scattered radiance. The total radiance is modeled with these components because they are readily obtained from MODTRAN5.

The proportions of these components vary significantly over the spectral region considered in this work as shown in Figures 2 and 3. Figure 2 shows top-of-atmospherespectral radiance, calculated with MODTRAN5, from RVPN on a typical day and Figure 3 gives a better idea of the fractional amount of each component. The near zero signal in the spectral regions near $1350 \mathrm{~nm}$ and $1800 \mathrm{~nm}$ are due to atmospheric water vapor absorption. Additional atmospheric absorbers as well as the inherent solar irradiance cause other sharply varying features in the radiance spectrum. Important features of 
Figure 3 to note are the rapidly increasing proportions of diffuse radiance components as shorter wavelengths are approached.

The goal is to predict the surface reflectance at the spectral locations covered by MODIS using radiance values reported by MODIS as values for $\mathrm{L}_{\text {total }}$. Unfortunately, surface reflectance is coupled with all three radiance components that make up the total at-sensor radiance shown in equation 1. One could rely on the appropriate data product from MODIS that provides the surface reflectance, but the goal of this effort is to develop a method that would be appropriate in the case that no such data product is available. $\mathrm{L}_{\text {diffuse }}$ is the smallest component of the total radiance throughout the spectrum. $\mathrm{L}_{\text {sky }}$ only becomes a significant portion of the total as the lower wavelength bound is approached. $\mathrm{L}_{\text {direct }}$ is the component that is most heavily dependent on surface reflectance for the spectral region longer than $500 \mathrm{~nm}$. Therefore, an approximation of surface reflectance can be made by modeling $\mathrm{L}_{\text {direct }}$ with

$$
L_{\text {direct }}=\frac{\rho_{\text {surface }} T_{\text {down }} T_{\text {up }} E_{\text {sun }} \cos \theta_{\text {SZA }}}{\pi} \quad \text { Eqn. } 2
$$

where $\rho_{\text {surface }}$ is surface reflectance, $T_{\text {down }}$ is the atmospheric transmission along the solarground path, Tup is the transmission along the ground-sensor path, $\mathrm{E}_{\mathrm{sun}}$ is exoatmospheric irradiance corrected for Earth-sun distance, and $\theta_{\mathrm{SZA}}$ is the solar zenith angle. This model assumes a Lambertian surface. The combination of equations 1 and 2 yields 


$$
\rho_{\text {surface }}=\frac{\pi\left(L_{\text {total }}-L_{\text {diffuse }}-L_{\text {sky }}\right)}{T_{\text {down }} T_{\text {up }} E_{\text {sun }} \cos \theta_{\text {SZA }}} \quad \text { Eqn. } 3
$$

An iterative algorithm is developed because inputs to MODTRAN require information about the surface reflectance, the desired product. The seed provided to the algorithm for surface reflectance input is the historical mean of RVPN. Atmospheric inputs to MODTRAN consist of the default desert aerosol model and average parameters found for RVPN. Previous work shows that MODTRAN inputs representing the average atmosphere have insignificant effects on calibration results relative to using atmospheric parameters derived for the given day when considering results averaged over multiple days (Leisso and Thome, 2006).

Outputs from the initial MODTRAN iteration include $\mathrm{L}_{\text {diffuse }}, \mathrm{L}_{\mathrm{sky}}, \mathrm{T}_{\mathrm{down}}$, and Tup. These are combined with spectral radiance reported by MODIS ( $\left.\mathrm{L}_{\text {total }}\right)$ to arrive at an initial estimate of multispectral surface reflectance according to equation 3 . These data are compared against the input reflectance that has been band averaged. A spectrally flat offset is found that minimizes the root mean square difference between the input reflectance and MODIS-derived reflectance according to

$$
R M S_{\text {difference }}=\sqrt{\frac{1}{N} \sum_{k}^{N}\left[\rho_{k, \text { MODIS-derived }}-\frac{\int_{\lambda} \rho_{k, \text { input }} R S R_{k} d \lambda}{\int_{\lambda} R S R_{k} d \lambda}+r_{\text {offset }}\right]^{2}} \text { Eqn. } 4
$$

where $\mathrm{N}$ is the number of MODIS bands, $\mathrm{k}$ is band number, $\rho_{\mathrm{k}}$, MODIS-derived is the surface reflectance at band $\mathrm{k}$ derived using MODIS radiance, $\rho_{\mathrm{k}}$, input is the reflectance 
that was input to MODTRAN, $\mathrm{RSR}_{\mathrm{k}}$ is the relative spectral response of MODIS band $\mathrm{k}$, and $r_{\text {offset }}$ is the offset that minimizes $R M S_{\text {difference. The algorithm applies }} r_{\text {offset }}$ to $\rho_{k}$, input and uses the result as the surface reflectance input for the next iteration. $r_{\text {offset }}$ typically converges to and insignificant amount after the algorithm completes six iterations.

Example results of the iterative algorithm are shown for 27 October 2006 and 7 May 2007 in Figure 3.14. The black line represents the historical average reflectance that served as the input seed to the algorithm. Six iterations resulted in the derived multispectral reflectance shown as the square and circle markers. The final reflectance products are shown as the blue and red lines which represent the reflectance curves of the test site that agrees best with multispectral reflectance derived from the well-understood MODIS sensor.

\section{Full spectrum at-sensor radiance}

The inter-calibration is applied for each Hyperion-MODIS image pair found. The final step before an inter-calibration comparison is to predict the at-sensor radiance for Hyperion. This is computed with MODTRAN using MODIS-derived surface reflectance found using the algorithm, described above, as a constraint. Atmospheric parameters used as inputs for MODTRAN are the same as those assumed in the reflectance algorithm: default desert aerosol model and average parameters of RVPN. This is similar to the reflectance-based approach but the reflectance spectrum is based on measurements from a satellite rather than ground-based spectrometer and atmospheric parameter means are 
assumed rather than coincident measurements with a solar radiometer. The next section presents results found with the inter-calibration method described here.

\section{Results}

The only parameters required for the inter-calibration method are the Hyperion and MODIS imagery and historically-averaged atmospheric and surface reflectance datasets. Therefore, any imagery of the test sites acquired by both sensors is available for use in inter-calibration which is dissimilar to the reflectance-based approach where a field campaign corresponds to only a handful of sensor acquisitions. While there were nine successful field campaigns for Hyperion at RVPN in the 2000-2009 period, there are 57 Hyperion scenes, 3002 Terra MODIS scenes, and 2431 Aqua MODIS scenes of RVPN the from which the inter-calibration method can select. The small number of Hyperion images relative to MODIS is a result of both the small swath of Hyperion and the fact that Hyperion does not operate on a $100 \%$ duty cycle but rather a limited number of scenes per orbit. In fact, the 57 scenes for RVPN is indicative of the importance of that test site since this is one of the largest number of scenes available for a single location in the entire Hyperion archive.

Processing routines developed for this project automate the procedure of defining the pixels that make up the test sites, finding averages and standard deviations of the radiance values corresponding to the test sites, and recording ancillary data such as view and solar geometry. These data are exported to a database where they are made available to the image pairing algorithm discussed in the previous section. Image pairing is based 
on both temporal and geometrical restrictions. Allowing a larger difference between Hyperion and MODIS of the sensor view and solar illumination angles increases the number of image pairs available for inter-calibration but increases uncertainty in results due to BRDF effects. Greater temporal differences between Hyperion and MODIS image acquisitions also increases the number of image pairs but increases uncertainty in results due to surface and atmospheric changes.

The remainder of this section presents inter-calibration results for the coincident and non-coincident cases. Reflectance-based approach results are presented as a baseline to evaluate different inter-calibration scenarios. The first scenario relies only on a coincident inter-calibration dataset (strictly not coincident data, but with time differences of less than an hour) based on the nine dates shown for the reflectance-based results. This is followed by results from non-coincident image pairs based on Terra and Aqua MODIS using the Railroad Valley test site. The results are shown as percent difference according to Equation 5.

\section{A. Coincident image pair results}

There were nine successful reflectance-based calibration attempts where in situ measurements of surface reflectance and atmospherics parameters were collected as Hyperion imaged the RVPN test site (Biggar et al., 2003, McCorkel et al., In review). A summary of the measurements and derived parameters from the reflectance-based collections are shown in Table 1 with the associated acquisition date. These data are used to predict the at-sensor radiance so that the calibration of Hyperion can be characterized. 
The results presented here are in the form of percent difference between radiance values of Hyperion and the prediction provided by the reflectance-based approach having a functional form of

$$
\% \text { difference }=100 \cdot \frac{L_{\text {Reference, } \lambda}-L_{\text {Hyperion }, \lambda}}{L_{\text {Reference, } \lambda}} \quad \text { Eqn. } 5
$$

where $L_{\text {Reference, } \lambda}$ and $L_{\text {Hyperion, } \lambda}$ are the spectral radiance values from the reflectance-based approach and Hyperion, respectively. The averaged results from the nine field campaigns are shown in Figure 5. Spectral regions of large deviation from zero, such as 940, 1130, 1350 and $2400 \mathrm{~nm}$, are affected by strong water vapor absorption. A combination of lower signal for both Hyperion and the ground-based spectrometer coupled with high sensitivity to atmospheric characterization in these spectral regions leads to unreliable results. The remainder of the data in Figure 5 shows agreement between Hyperion and reflectance-based results to within $-5 \%$ and $+10 \%$. A convenient way to assess the consistency of these results is to find the standard deviation of the mean as shown in Figure 7. Excluding the high variability in spectral regions affected by water vapor absorption, the consistency for the reflectance-based method is on the $2 \%$ level. The band-to-band consistency of the standard deviation indicates that band-to-band variability in the percent differences are real effects caused by the calibration of Hyperion. The implication of these results is that a smoothly varying spectral radiance would display band-to-band variations on the order of 5-10\% simply because of the radiometric calibration of the system.

The results shown here for Hyperion compare favorably in standard deviation to those derived for other sensors (Thome, 2001; Thome et al., 2004a,b, McCorkel et al., 2006). Such favorable comparisons give confidence in the results for Hyperion. 
Additionally, each of the nine dates shown also included calibration attempts for other sensors using the ground data collected for Hyperion or using data sets collected near in time and location for other sensors. The results for the other sensors are consistent with long-term trends for those instruments (such as Landsat-5 Thematic Mapper and Landsat 7 Enhanced Thematic Mapper Plus). The internal consistency of the reflectance-based results with other dates and imagers gives good confidence in the average percent difference shown for Hyperion making this characterization an excellent baseline to compare against other calibration methods.

The most direct comparison between reflectance-based and inter-calibration results is made when considering identical dates in both datasets. All nine Hyperion scenes used in the reflectance-based results are paired with a Terra MODIS scene that was acquired approximately 30-40 minutes later. The overpass times and associated solar zenith angle are shown in Table 2.

The red line shown in Figure 5 shows mean results of the inter-calibration method for the case when dates identical to the reflectance-based method are used. Two features are clearly evident in the figure when comparing the cross-calibration results to the reflectance-based results. The first is that the shapes of the two curves appear to be highly correlated giving further indication that the band-to-band features seen in the figure are inherent to the radiometric calibration of Hyperion. The second feature that is evident is an apparent bias between the reflectance-based and cross-calibration results. Recall that the inter-calibration results are based on comparison to Terra MODIS. Thus, any bias between the reflectance-based method and the calibration of Terra MODIS would show 
up as a bias between the calibration of Hyperion using the two approaches. Such a bias has been shown to exist in other work (Thome et al., 2003).

Clearly, what is of interest would be any strong spectral differences between the reflectance-based and inter-calibration approach. Figure 6 shows percent difference between the reflectance-based and inter-calibration results and the lack of band-to-band features demonstrates that similar Hyperion spectral effects are present in both approaches. The effects of atmospheric absorption are evident in larger percent differences in spectral regions affected by absorption. The larger difference is a result both of temporal changes in water vapor content with time as well as poorer signal-tonoise effects. The shape of the percent difference indicates a bias, as described above, that is within $2 \%$ save spectral regions of strong atmospheric absorption. The curve is reasonably flat in non-absorption regions of the spectrum except at shorter wavelengths for which there is a slope to the difference curve. The cause of this feature is likely due to the shape of the spectral reflectance used in the inter-calibration approach. Recall that the inter-calibration approach uses a singular average spectral reflectance with a day-today correction for the overall brightness changes in the surface. These results are further indication that the spectral shape of at least a portion of the RVPN test site is relatively invariant with time, when dry surface conditions are present.

Comparing the variability of the datasets also illustrates the probable sources of uncertainty in the inter-calibration approach. The percent standard deviation of the mean percent difference for the reflectance-based and inter-calibration approaches is shown in Figure 7. The variability of the inter-calibration results is higher in most spectral regions. Likely causes of the larger standard deviation of the inter-calibration approach are 
atmospheric scattering effects, uncertainty in the spectral reflectance curve fit, and spatial registration of the MODIS data to the Hyperion data.

\section{B. Non-coincident image pair results}

Non-coincident image pair selection for inter-calibration may introduce uncertainty due to temporal atmospheric and surface variations, but allows comparison between sensors that do not share a similar orbit. As mentioned previously, larger differences in time and view-sun geometry that are allowed between the reference and test sensor lead to more possible comparison data sets. The work here for used a limit of 6 degrees for the difference in view and illumination angles between Hyperion and MODIS. A temporal difference between image acquisitions is limited to 30 days. These restrictions are chosen based on a compromise between processing resources and number of image pairs returned. Subsets of these restrictions such as tighter geometry constraints or shorter temporal difference return fewer image pairs and show minimal difference in final results.

The 6-degree geometry and 30-day temporal restrictions and subsequent intercalibration processing returns 80 and 82 successful image pairs for Hyperion-Terra MODIS and Hyperion-Aqua MODIS, respectively. It is common for a single Hyperion scene to be paired with multiple MODIS scenes, and this is an advantage to using the wide-swath multispectral sensors for inter-calibration of a narrow-swath sensor. 
Inter-calibration results are presented alongside those of the reflectance-based approach shown in Figure 8. The results obtained using different MODIS sensors are kept separate where the blue line is Terra-based and the green line is Aqua-based. The black line shows results for the reflectance-based and is shown here for reference. There is strong agreement between results that are based on different reference sensors. This gives confidence that the two MODIS sensors agree well with each other, but it also shows that a consistent calibration can be achieved by use of the particular MODIS sensor that provides the most convenient image pairing.

Of significant interest in the non-coincident data sets is how well they agree with the coincident data set. The lines in Figure 9 show percent difference between the intercalibration method and the reflectance-based approach for the coincident Terra MODIS (red line), non-coincident Terra MODIS (blue line), and non-coincident Aqua MODIS (green line) data sets. The similarity between the coincident and non-coincident data sets is encouraging. The shapes of the lines are consistent with each other: appearance of a bias, large percent differences near areas of strong atmospheric absorption, and consistent band-to-band features. The band-to-band features in the 2000-2400 nm region in Figure 9 are consistent among the Aqua- and Terra-based non-coincident data sets but are not seen in the Terra-based coincident data set. Although a small effect, reasons for this inconsistency are unclear.

Results based on non-coincident image pairs have strong agreement with the reflectance-based and coincident inter-calibration data sets; however, differences in the variability of the data sets are shown by their standard deviations in Figure 10. The noncoincident datasets have variability that is about 3-4 times that of the reflectance-based 
approach. Similarities in calibration results, but with higher variability, relative to the reflectance-based approach suggest that BRDF and temporal changes at the test site average out over large datasets.

One objective of sensor characterization is to assess sensor degradation. Figure 11 shows a time series of Aqua MODIS-based inter-calibration of Hyperion using noncoincident image pairs. Two channels are shown, one for each focal plane of Hyperion: $32(671 \mathrm{~nm})$ and $145(1598 \mathrm{~nm})$. Although there is much scatter there is no distinguishable trend. This is consistent with studies using lunar acquisitions that find Hyperion has been stable over its lifetime.

Transferring the absolute calibration from a wide-swath sensor to a narrow-swath imager is highly flexible with the inter-calibration method developed here. As mentioned above this study uses Hyperion-MODIS image pairing restricted to 6-degree geometry and 30-day temporal differences. Figure 12 shows a histogram of image acquisition date separation between Hyperion and MODIS. Shorter and longer temporal difference restrictions decreased and increased the number of image pairs, but showed little difference in calibration and variability.

\section{Conclusion}

An inter-calibration method is developed that provides absolute radiometric calibration of a narrow-swath imager with reference to non-coincident wide-swath sensor. This work uses the well-understood calibration of MODIS as a reference to find the spectral reflectance of a test site with a simple iterative radiative transfer method and 
spectral shape information based on historical ground-based measurements. The imagery of the reference sensor is restricted to acquisitions that provide similar view and solar illumination geometry to reduce uncertainties due to directional reflectance effects. Atsensor radiance is calculated for the narrow-swath sensor, Hyperion, using this spectral reflectance and atmospheric parameters that are also based on historical in situ measurements.

Calibration results for the Hyperion sensor are presented for three data sets: reflectance-based approach based on ground reflectance and atmospheric measurements, inter-calibration using coincidently acquired imagery, and inter-calibration using noncoincidently acquired imagery. Past work for Hyperion has shown reflectance-based results for with $2 \%$ repeatability and $3-5 \%$ uncertainty depending on spectral region. These data are used as a baseline to assess the agreement of the inter-calibration results. Inter-calibration results based on coincident image pairs and using the same image dates agree with reflectance-based result to the $2 \%$ level with $2-5 \%$ repeatability depending on spectral region. They are highly correlated giving further indication that the band-to-band features seen in both set of results are inherent to the radiometric calibration of Hyperion.

Non-coincident inter-calibration data sets agree well with reflectance-based and coincident inter-calibration results, but have increased variability. Also, non-coincident Terra MODIS-based and Aqua MODIS-based inter-calibration results are found to be highly consistent with each other providing confidence that the two MODIS sensors compare with each other and shows that a consistent calibration can be achieved by use of the particular MODIS sensor that provides the most convenient image pairing. The 
method developed here enables inter-calibration between recent and upcoming climategrade sensors. 


\section{References}

Arai, K., Tonooka, H., "Radiometric performance evaluation of ASTER VNIR, SWIR, and TIR," IEEE Transactions of Geoscience and Remote Sensing 43(12), 2725-2732 (2005).

Barnes, R. A., R. E. Eplee, Jr., G. M. Schmidt, F. S. Patt, and C. R. McClain, “Calibration of SeaWiFS. I. Direct Techniques," Applied Optics, 40, pp. 6682-6700, 2001.

Biggar, S. F., K. J. Thome, W. T. Wisniewski, "Vicarious radiometric calibration of EO1 sensors by reference to high-reflectance ground targets," IEEE Trans. On Geosciences and Remote Sensing, 41, pp. 1174-1179, 2003.

Chander, G., D.J. Meyer, D.L. Helder, "Cross calibration of the Landsat-7 ETM+ and E0-1 ALI Sensor," IEEE Trans. on Geosci. and Remote Sensing, Vol. 42, No. 12, pp. 2821- 2831, 2004.

Cabot, F., O. Hagolle, and P. Henry, "Relative and multitemporal calibration of AVHRR, SeaWiFS, and VEGETATION using POLDER characterization of desert sites," Geoscience and Remote Sensing Symposium Proceedings, Vol. 5, pp. 2188-2190, 2000.

Heidinger, A.K., C. Cao, and J. T. Sullivan, "Using Moderate Resolution Imaging Spectrometer (MODIS) to calibrate advance very high resolution radiometer reflectance channels, Journal of Geophys. Res, Vol. 107, pp. 4702-4704, 2002.

Leisso, N., K. Thome, "Effect of atmospheric characterization on the reflectance based vicarious method of radiometric calibration of Landsat 7 ETM+", Proc. 15th Annual CALCON Technical Conference, Logan, Utah, 2005.

McCorkel, J. K.T. Thome, S.F. Biggar, M.J. Kuester, "Radiometric Calibration of Advanced Land Imager using reflectance-based results between 2001 and 2005," Proceedings of SPIE, Vol. 6296, 2006.

McCorkel, J., K.J. Thome, L. Ong, "Vicarious calibration EO-1 Hyperion,” IEEE Journal of Selected Topics in Applied Earth Observations and Remote Sensing, In review.

Nguyen, L., D.R. Doelling, P. Minnis, J.K. Ayers, "Rapid technique to cross calibrate satellite imager visible channels,” Proc. SPIE, Vol. 5542, pp 227-235, 2004.

O’Brien, D.M., R.M. Mitchell, “An error budget for cross-calibration of AVHRR shortwave channels against ASTR-2," Remote Sensing of Enviroment, Vol. 75, No. 2, pp. 216-229, 2001.

Scott, K. P., Thome, K. J., Brownlee, M. R., "Evaluation of the Railroad Valley playa for use in vicarious calibration,” Proc. SPIE 2818, 158-166 (1996). 
Teillet, P.M., P.N. Slater, Y. Ding, R.P. Santer, R.D. Jackson, M.S. Moran, “Three methods for the absolute calibration of the NOAA AVHRR sensors in-flight," Remote Sensing of Environment, Vol. 31, No 2., pp. 105-120, 1990.

Teillet, P.M., J.L Barker, B.L Markham, R.R. Irish, G. Fedosejevs, J.C. Storey, "Radiometric cross-calibration of the Landsat-7 ETM+ and Landsat-5 TM sensors based on tandem data sets," Remote Sensing of Environment, Vol. 78, No. 1, pp. 3954, 2001a.

Teillet, P. M., G. Fedosejevs, R. P. Gauthier, N. T. O’Neill, K. J. Thome, S. F. Biggar, H. Ripley, and A. Meygret, "A generalized approach to the vicarious calibration of multiple Earth observation sensors using hyperspectral data," Remote Sensing of Environment, 77, pp. 304-327, 2001b.

Teillet, P.M., B.L. Markham, and R.R. Irish, "Landsat cross-calibration based on near simultaneous imaging of common ground targets," Remote Sensing of Environment, Vol. 102, No. 3-4, pp. 264-270, 2006.

Teillet, P.M., G. Fedosejevs, K.J. Thome, J.L. Barker, “Impacts of spectral band difference on radiometric cross-calibration between satellite sensors in the solar reflective sprectral domain," Remote Sensing of Environment, Vol. 110, No. 3, pp. 393-409, 2007.

Thome, K. J., "Absolute radiometric calibration of Landsat 7 ETM+ using the reflectance- based method," Remote Sensing of Environment 78(1), 27-38, 2001.

Thome, K.J., E.E. Whittington, and N. Smith, "Radiometric calibration of MODIS with reference to Landsat-7 ETM+," Proceedings of SPIE, Vol. 4483, pp. 203-210, 2003.

Thome, K., S. Biggar, and H. J. Choi, "Vicarious calibration of Terra ASTER, MISR, and MODIS,” Proc. SPIE Conf. \#5542, pp. 290-299, Denver, Colo., 2004.

Thome, K. J., Helder, D. L., Aaron, D., Dewald, J. D., "Landsat-5 TM and Landsat-7 ETM+ Absolute Radiometric Calibration Using the Reflectance-Based Method," IEEE Transactions of Geoscience and Remote Sensing 42(12), 2777-2785, 2004.

Thome, K.J.; Arai, K.; Tsuchida, S.; Biggar, S.F., "Vicarious Calibration of ASTER via the Reflectance-Based Approach," IEEE Transactions of Geoscience and Remote Sensing 42, 46 (10), 3285-3295, 2008.

Trischenko, A.P., J. Cihlar, Z. Li, "Effects of spectral response function on surface reflectance and NDVI measured with moderate resolution satellite sensors," Remote Sensing of Environment, Vol. 81, No. 1, pp. 1-18, 2002. 
Vane, G., Green, R. O., Chrien, T. G., Enmark, H. T., Hansen, E. G., Porter, W. M., “The Airborne Visible/Infrared Imaging Spectrometer (AVIRIS)," Remote Sensing of Environment 44, 127-143 (1993).

Xiong, X., Chiang, K., Esposito, J., Guenther, B., and Barnes, W.L., 2003, “MODIS Onorbit Calibration and Characterization", Metrologia 40, 89-92.

Xiong, X., W. Barnes, “Earth Science Satellite Remote Sensing,” Springer, Vol. 2, 2006. McCorkel, J., K.J. Thome, "Vicarious calibration of Hyperion," Journal of Selected Topics in Applied Earth Observations and Remote Sensing, Submitted for publication.

Wu, A., X. Xiong, and C. Cao, "Terra and Aqua MODIS inter-comparison of three reflective solar bands using AVHRR onboard the NOAA-KLM satellites," Int'l. Journal of Rem. Sens.Vol.29, pp. 1997-2010, 2008.

Joel McCorkel received a BS degree in Optical Engineering and PhD in Optical Sciences from the University of Arizona. He joined the National Ecological Observatory Network as a staff scientist for the Airborne Observation Platform in 2009. He is currently a physical scientist in the Biospheric Sciences Laboratory at NASA's Goddard Space Flight Center, Greenbelt, Maryland.

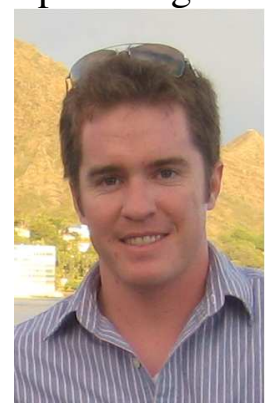

Kurt Thome obtained a BS degree in meteorology from Texas A\&M University and MS and $\mathrm{PhD}$ degrees in atmospheric sciences from the University of Arizona. He then joined what is now the College of Optical Sciences becoming full professor in 2006. He served as the Director of the Remote Sensing Group from 1997 to 2008. Thome moved to NASA's Goddard Space Flight Center in 2008 as a Physical Scientist in the Biospheric Sciences Laboratory. He has been a member of the Landsat-7, ASTER, MODIS, and EO-1 Science Teams providing vicarious calibration results for those and other imaging sensors. He is a Fellow of SPIE, is the Instrument Scientist for the Visible Infrared Imaging Radiometer Suite on the Joint Polar Satellite System and is serving as the calibration lead for the Thermal Infrared Sensor on the Landsat Data Continuity Mission. Thome is the Deputy Project Scientist for CLARREO for which he is also the instrument lead for the Reflected Solar Instrument. 


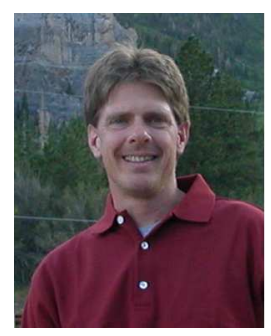

Ronald B. Lockwood received a BA degree from Texas Tech University and MS and $\mathrm{PhD}$ degrees from the University of Wisconsin at Madison, all in physics. He is a member of the technical staff at MIT Lincoln Laboratory, in Lexington, Massachusetts. Previously, from 1993 through 2009, he worked in the Space Vehicles Directorate and its predecessor organizations at the Air Force Research Laboratory at Hanscom AFB, MA. He was the Deputy Principal Investigator for the ARTEMIS payload, a space-based imaging spectrometer, where his work focused on the sensor calibration and performance. He continues to develop technology for spectral remote sensing applications at MIT Lincoln Laboratory.

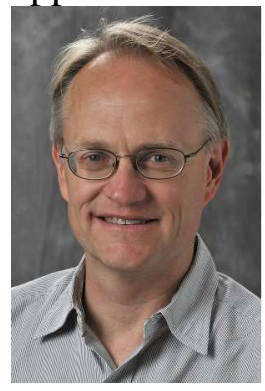




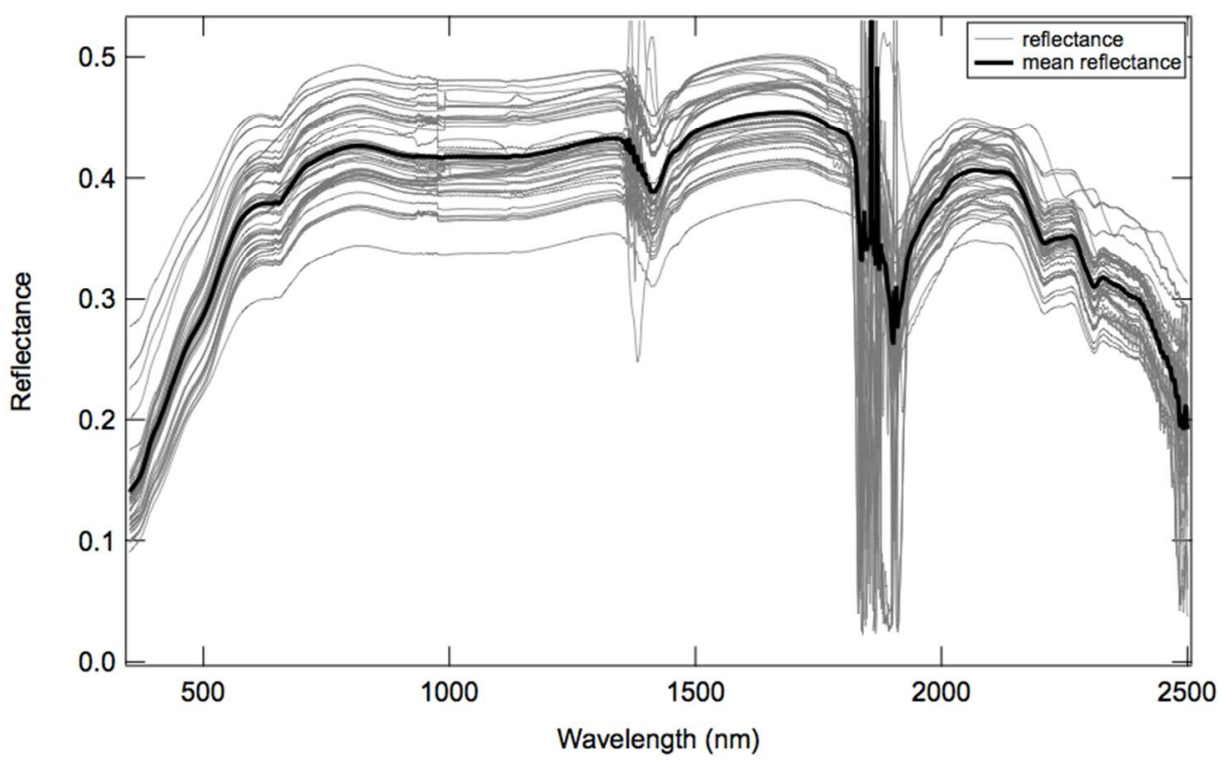

Fig. 1. Surface reflectance of the test site at RVPN. The thin lines represent data retrieved over the 2000-2009 period and the black line is the mean of the historical reflectance.

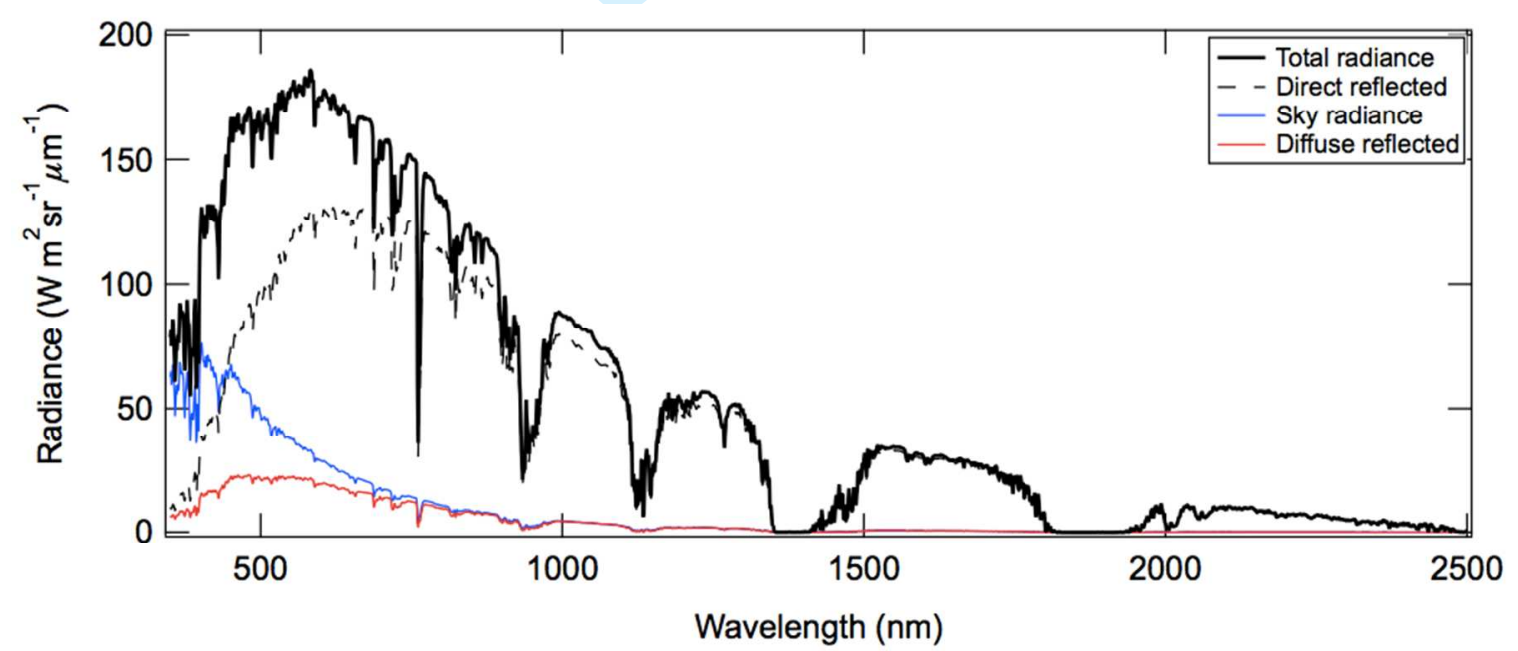

Fig. 2. Top-of-atmosphere total and component spectral radiance for a typical day at RVPN. 


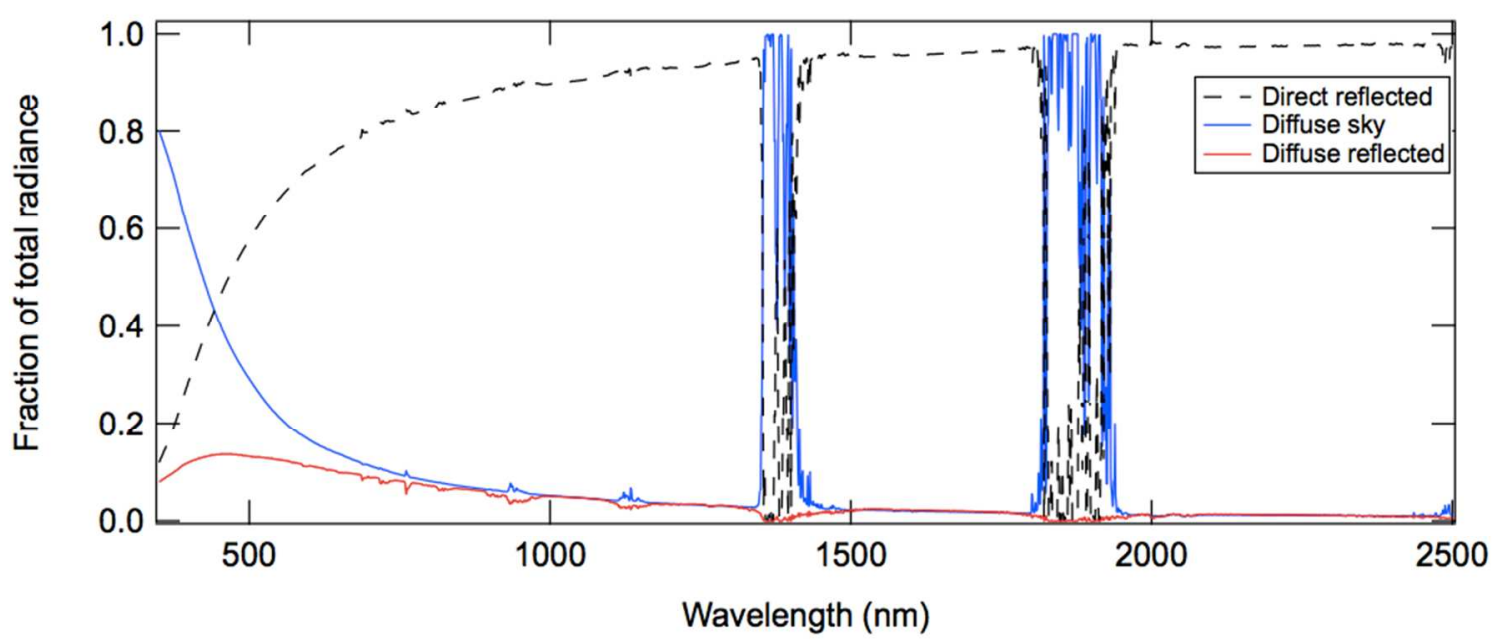

Fig. 3. Fractional components of top-of-atmosphere spectral radiance for a typical day at RVPN.

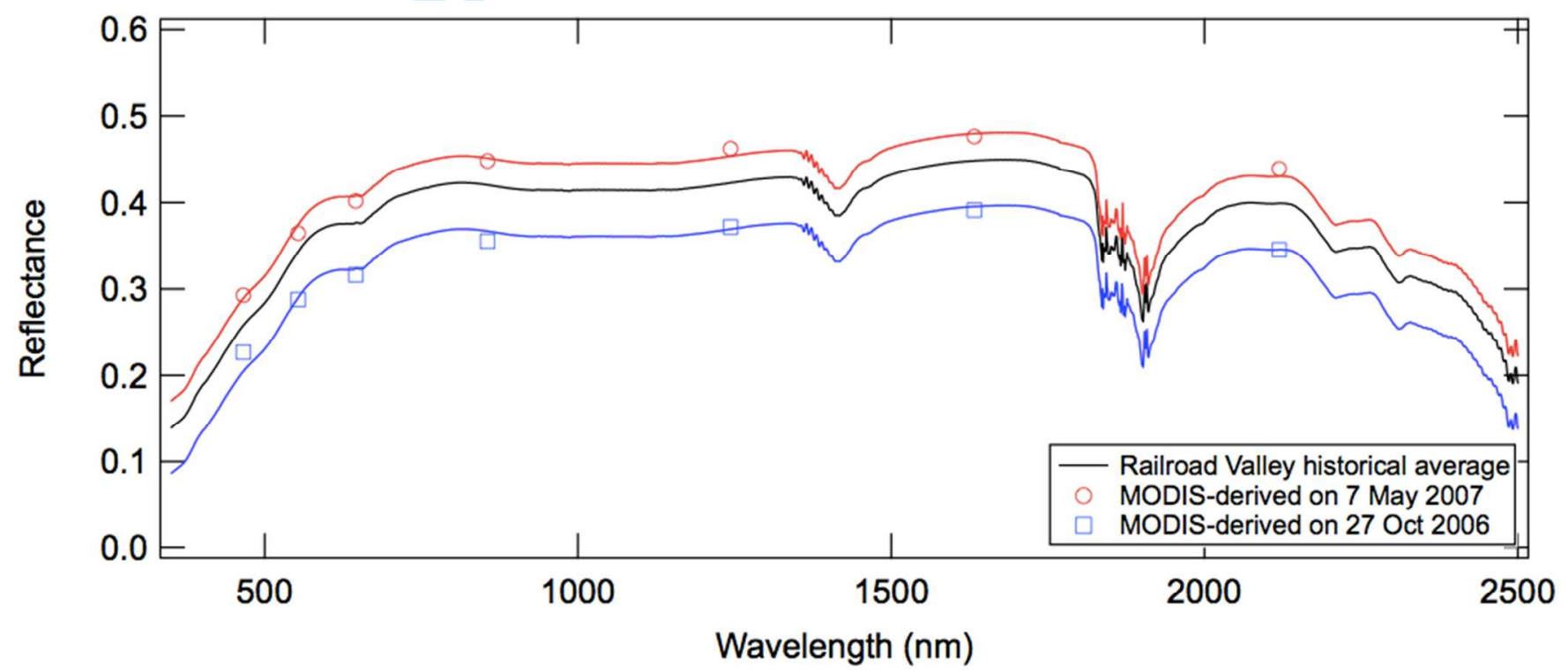

Fig. 4. Surface reflectance of RVPN on 27 October 2006 and 7 May 2007. The markers show multispectral reflectance derived using MODIS radiance, the black line is the mean of historical in situ measurements, and the blue and red lines are equal to the mean plus an offset that minimizes the difference relative to the markers. 


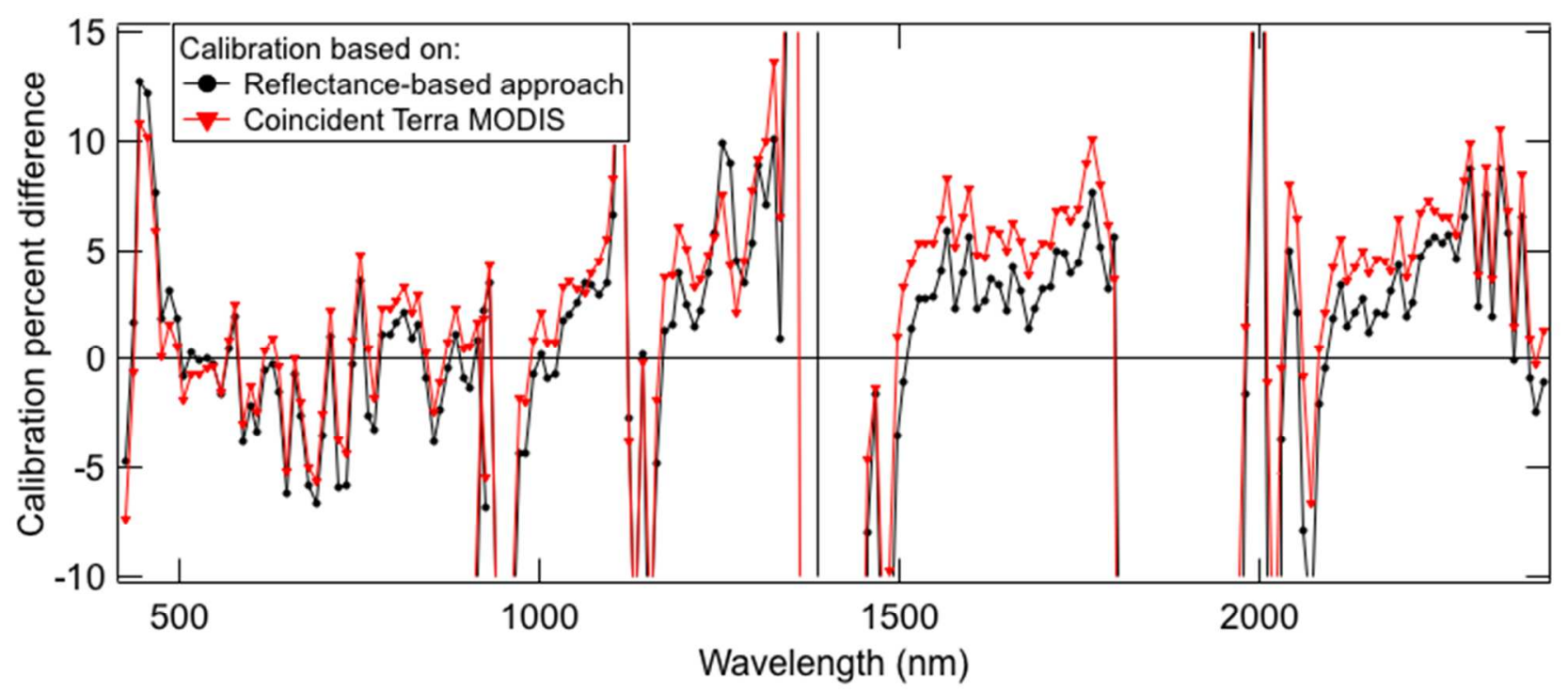

Fig. 5. Comparison of radiance reported by Hyperion and predicted radiance. The black line shows the mean results of the reflectance-based approach and the red line shows mean results of the inter-calibration method using the same set acquisition dates.

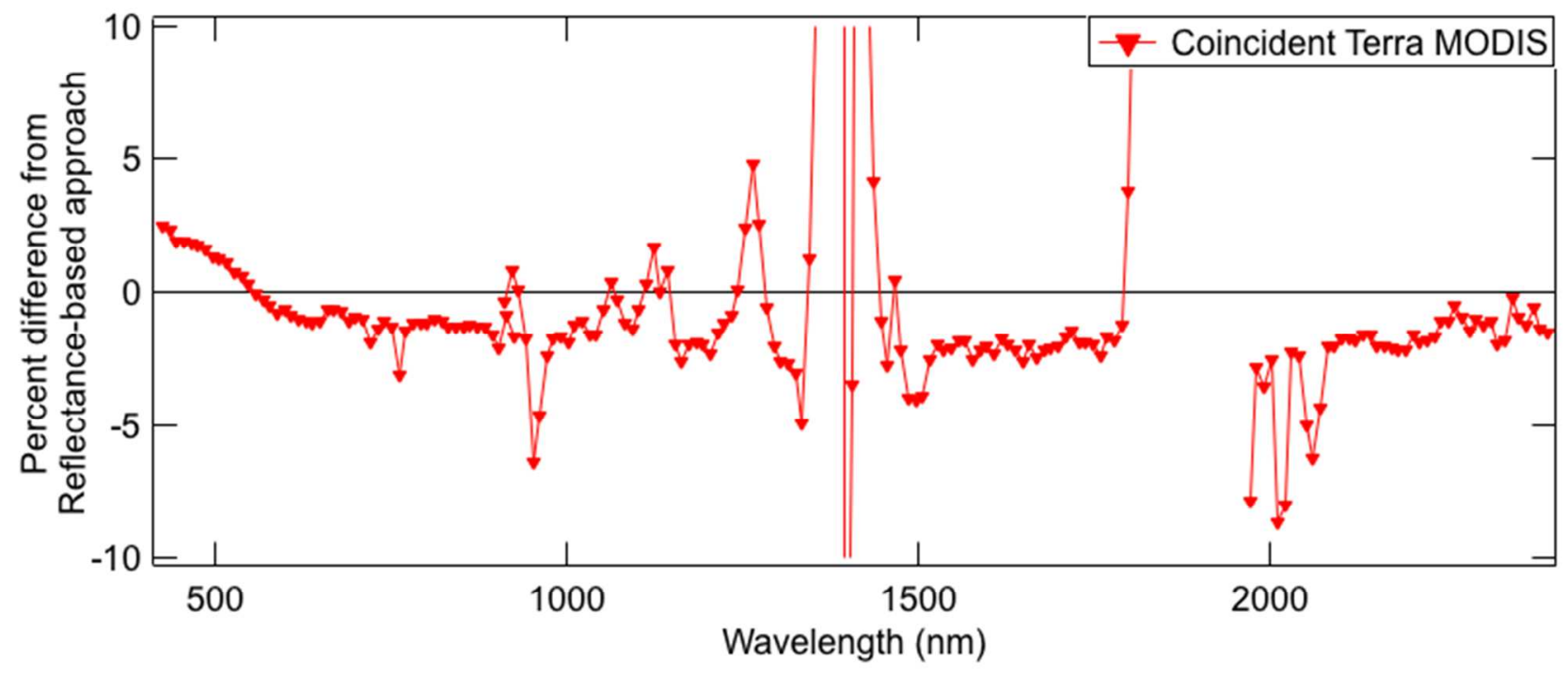

Fig. 6. Percent difference between mean results from the reflectance-based approach and inter-calibration method based on identical dates for both approaches. 


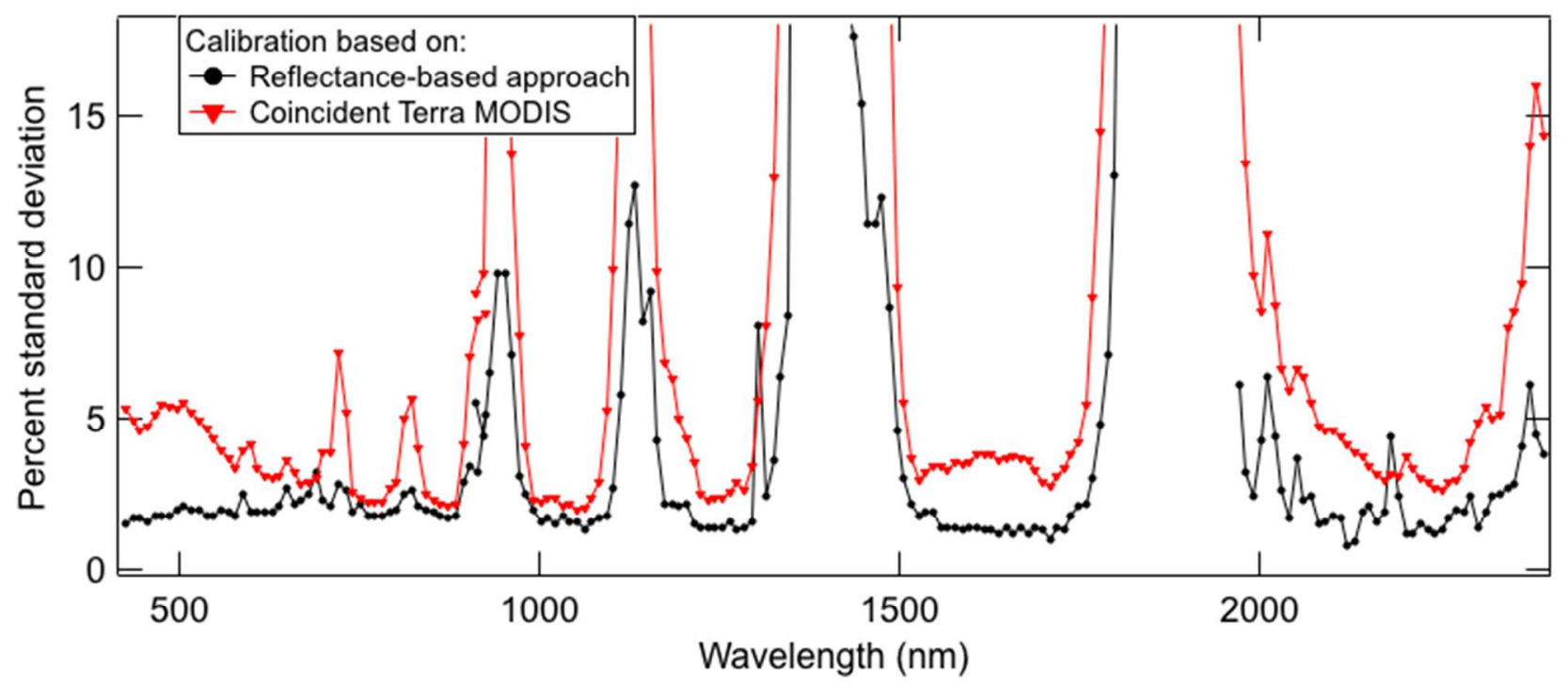

Fig. 7. Standard deviation associated with the mean results of the reflectance-based approach and inter-calibration method using the coincident imagery pairs.

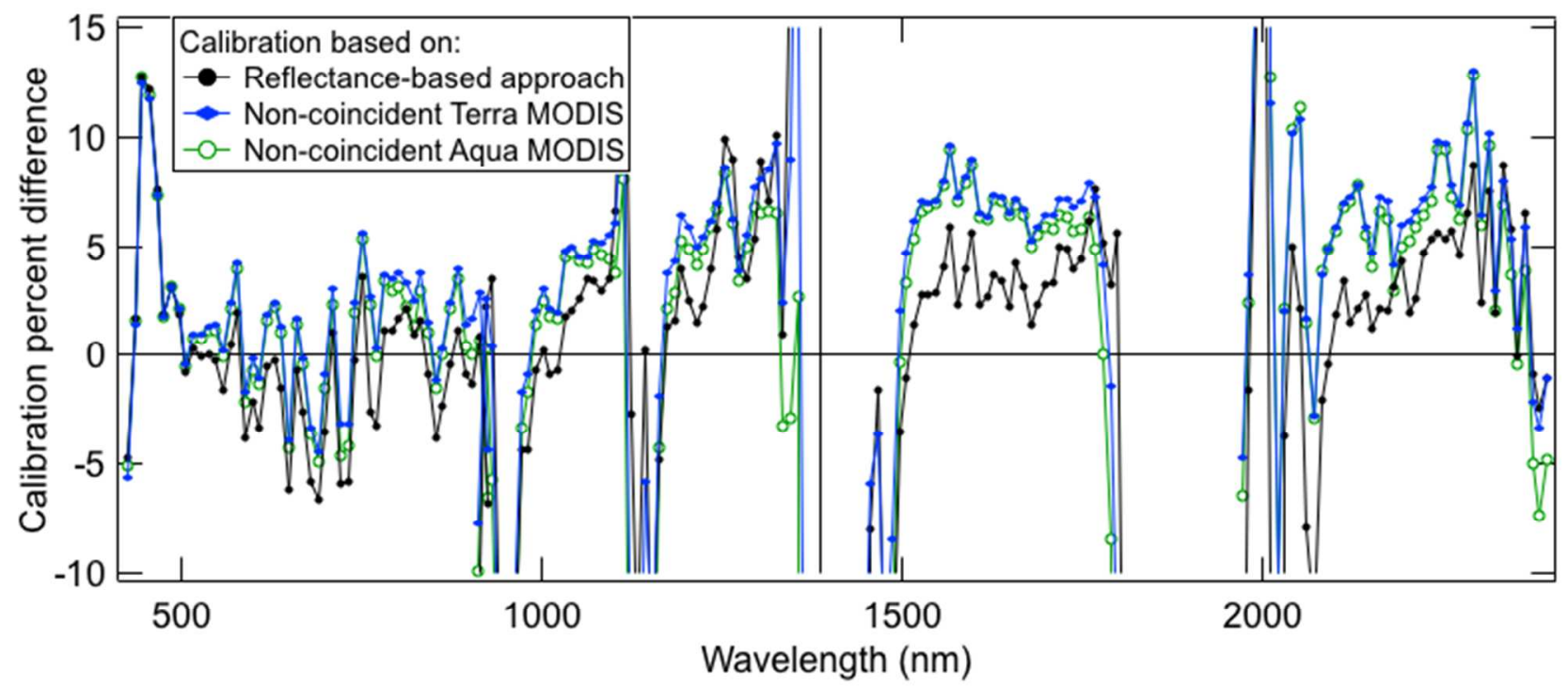

Fig. 8. Comparison of radiance reported by Hyperion and predicted radiance. The black line shows the mean results of the reflectance-based approach and the blue and green lines show mean results of the inter-calibration method using non-coincident image pairs with Terra MODIS and Aqua MODIS, respectively. 


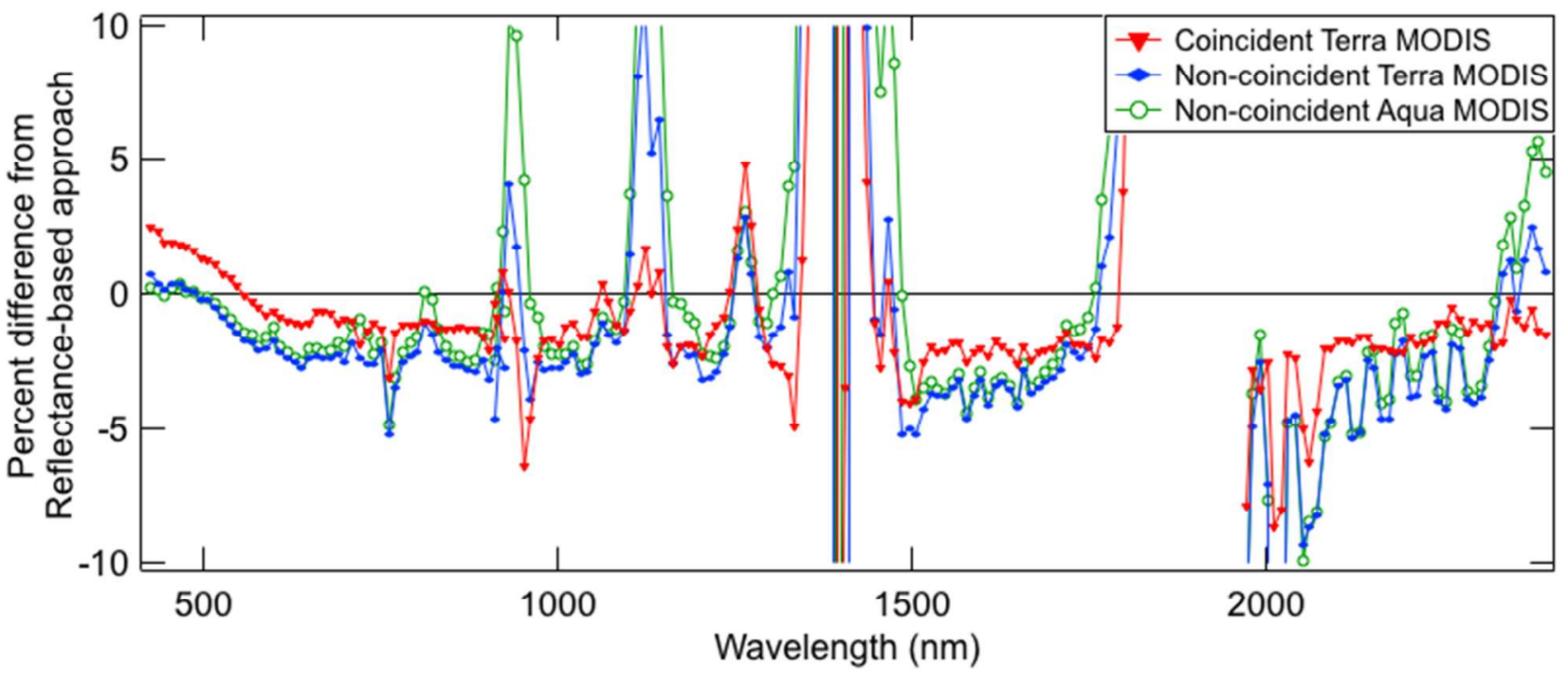

Fig. 9. Percent difference between results of the reflectance-based approach and the intercalibration method using coincident Terra MODIS, non-coincident Terra MODIS, and non-coincident Aqua MODIS image pairing with Hyperion.

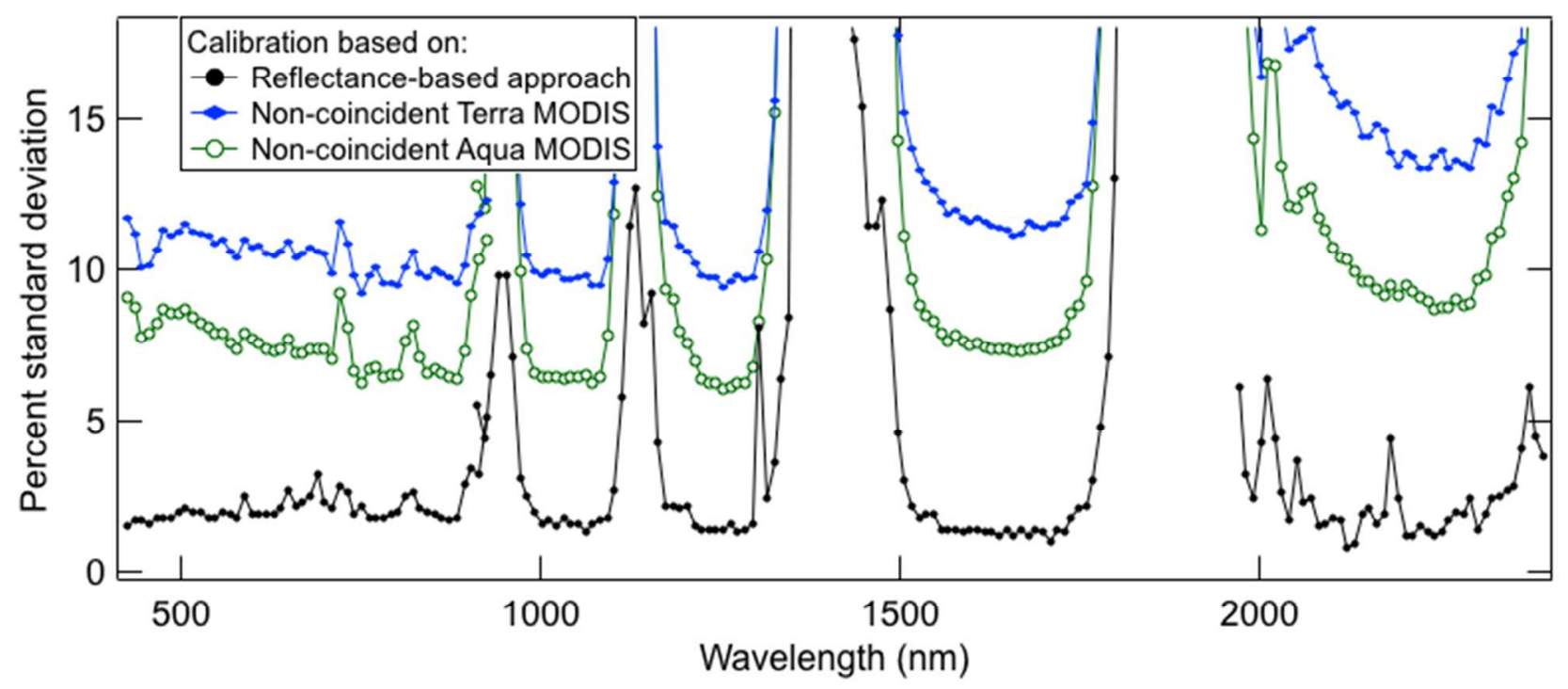

Fig. 10. Standard deviation associated with the mean results of the reflectance-based approach and inter-calibration method using the non-coincident image pairs. 


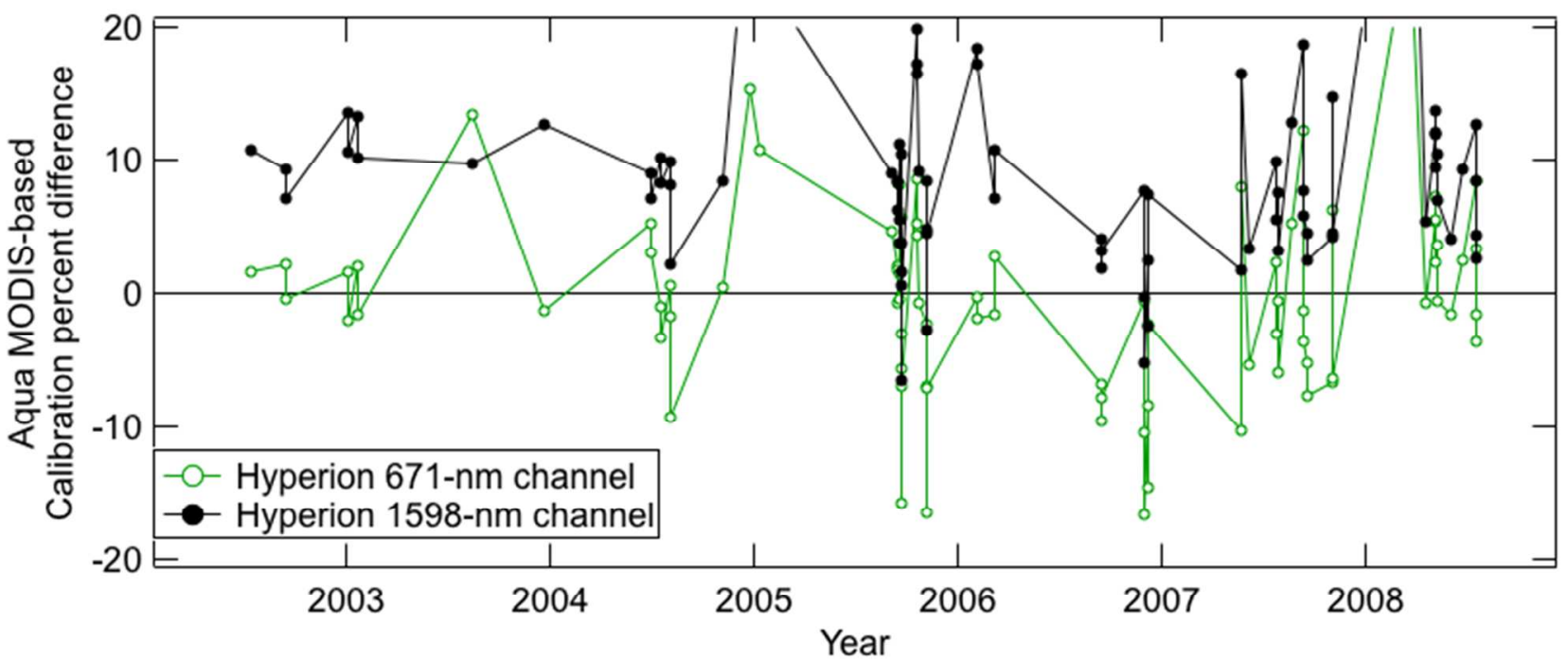

Fig. 11. Inter-calibration times series for Hyperion channels 32 (671-nm) and 145 (1598$\mathrm{nm})$.

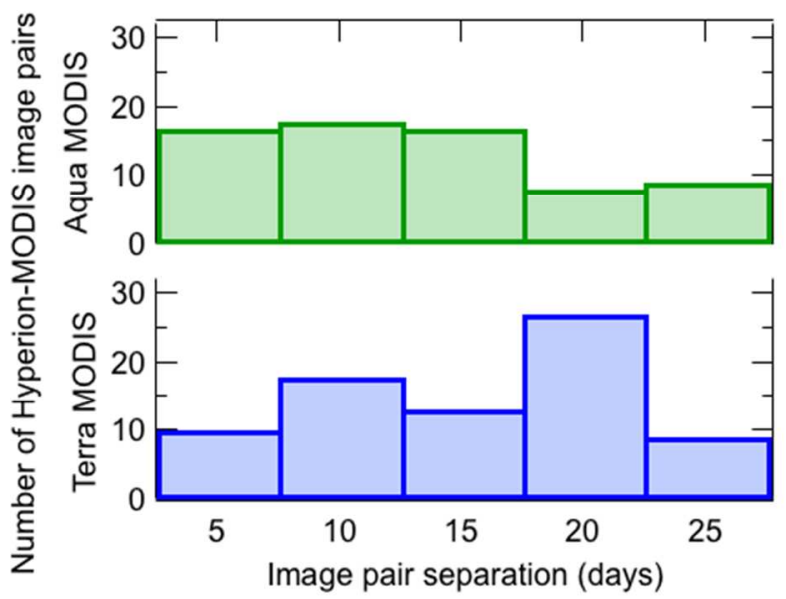

Fig. 12. Frequency of Hyperion-MODIS image pairs used in non-coincident intercalibration versus date separation. 
Table I. Summary of measurement data for the nine Hyperion acquisitions of RVPN with coincident ground collections.

\begin{tabular}{|c|c|c|c|c|c|c|c|c|c|}
\hline Acquisition date & $\begin{array}{r}13 \text { May } \\
2001 \\
\end{array}$ & $\begin{array}{r}14 \text { June } \\
2001 \\
\end{array}$ & $\begin{array}{r}16 \text { July } \\
2001 \\
\end{array}$ & $\begin{array}{r}17 \text { June } \\
2002 \\
\end{array}$ & $\begin{array}{r}22 \text { July } \\
2003 \\
\end{array}$ & $\begin{array}{r}18 \text { March } \\
2004 \\
\end{array}$ & $\begin{array}{r}22 \text { June } \\
2004 \\
\end{array}$ & $\begin{array}{r}\text { July } \\
2004 \\
\end{array}$ & $\begin{array}{r}5 \text { March } \\
2005 \\
\end{array}$ \\
\hline Acquisition time (UTC) & $18: 12: 04$ & $18: 11: 40$ & $18: 11: 24$ & $18: 10: 34$ & $18: 10: 37$ & $18: 11: 20$ & $18: 11: 10$ & 18.10:59 & 18:11:50 \\
\hline Surface refl time (UTC) & $\begin{array}{l}18: 13- \\
18: 42\end{array}$ & $\begin{array}{r}17: 50- \\
18: 22\end{array}$ & $\begin{array}{l}17: 40- \\
18: 23\end{array}$ & $\begin{array}{r}17: 33- \\
18: 10\end{array}$ & $\begin{array}{c}17: 46- \\
17: 53\end{array}$ & $\begin{array}{c}17: 45- \\
18: 22\end{array}$ & $\begin{array}{c}17: 37- \\
18: 15\end{array}$ & $\begin{array}{r}17: 35- \\
18: 11\end{array}$ & $\begin{array}{r}17: 36- \\
18: 14\end{array}$ \\
\hline Solar zenith range & $27.2-23.4$ & $28.3-22.8$ & $32.5-25.1$ & $31.4-24.9$ & $32.1-30.8$ & $48.5-43.9$ & $31.0-24.3$ & $32.6-26.1$ & $54.6-49.9$ \\
\hline Test site layout $\mathrm{m}^{2}$ & $90 \times 240$ & $90 \times 240$ & $90 \times 240$ & $120 \times 480$ & $120 \times 480$ & $120 \times 480$ & $120 \times 480$ & $120 \times 480$ & $120 \times 480$ \\
\hline Solar zenith & 27.4 & 24.6 & 27.0 & 24.8 & 28.0 & 45.3 & 24.9 & 26.1 & 50.4 \\
\hline Solar azimuth & 130.6 & 121.6 & 122.8 & 120.8 & 123.6 & 143.8 & 120.6 & 121.3 & 146.0 \\
\hline View zenith & 1.6 & 1.4 & 1.5 & 1.3 & 0.3 & 1.4 & 1.3 & 1.3 & 0.1 \\
\hline View azimuth & 98.2 & 98.2 & 98.2 & 98.2 & 103.0 & 98.2 & 98.2 & 98.2 & 105.0 \\
\hline Temperature (C) & 32 & 13 & 30 & 34 & 39 & 19 & 30 & 33 & 13 \\
\hline Pressure (mbar) & 858 & 855 & 851 & 856 & 859 & 859 & 857 & 854 & 860 \\
\hline Angstrom parameter & 1.16 & 1.68 & 0.35 & 1.12 & 1.42 & 0.90 & 2.18 & 1.03 & 0.66 \\
\hline Water vapor (cm) & 1.36 & 0.53 & 0.77 & 0.56 & 2.48 & 0.76 & 1.47 & 1.71 & 0.91 \\
\hline $\begin{array}{l}\text { Aerosol optical depth } \\
\text { @ } 550 \mathrm{~nm}\end{array}$ & 0.073 & 0.032 & 0.040 & 0.110 & 0.097 & 0.075 & 0.095 & 0.089 & 0.038 \\
\hline Ozone (DU) & 308 & 311 & 328 & 303 & 280 & 313 & 296 & 299 & 308 \\
\hline
\end{tabular}

Table II. Coincident Hyperion and Terra MODIS image pairs

\begin{tabular}{ccccccc}
\hline & \multicolumn{2}{c}{ Overpass time } & \multicolumn{2}{c}{ View zenith } & \multicolumn{2}{c}{ Solar zenith } \\
\cline { 2 - 7 } Acquisition date & Hyperion & Terra MODIS & Hyperion & Terra MODIS & Hyperion & Terra MODIS \\
\hline $2001-05-13$ & $18: 12: 04$ & $18: 48: 14$ & 0.2 & 0.1 & 27.5 & 22.8 \\
$2001-06-14$ & $18: 11: 40$ & $18: 47: 21$ & 0.1 & 0.5 & 24.7 & 19.3 \\
$2001-07-16$ & $18: 11: 24$ & $18: 46: 41$ & 0.2 & 0.3 & 27.2 & 21.8 \\
$2002-06-17$ & $18: 10: 34$ & $18: 40: 05$ & 0.1 & 0.6 & 24.9 & 20.3 \\
$2003-07-22$ & $18: 10: 37$ & $18: 37: 41$ & 0.1 & 1.5 & 28.0 & 23.9 \\
$2004-03-18$ & $18: 11: 20$ & $18: 38: 53$ & 0.1 & 0.7 & 45.2 & 42.4 \\
$2004-06-22$ & $18: 11: 10$ & $18: 38: 19$ & 0.1 & 0.8 & 25.0 & 20.7 \\
$2004-07-08$ & $18: 10: 59$ & $18: 38: 20$ & 0.1 & 0.6 & 26.2 & 21.9 \\
$2005-03-05$ & $18: 11: 50$ & $18: 37: 51$ & 0.1 & 1.0 & 50.3 & 47.7 \\
\hline
\end{tabular}

